\title{
Kindgerechte Erkältungsmedizin
}

— Nicht jedes zur Selbstmedikation in der Apotheke angebotene Erkältungsmittel ist auch für Kinder geeignet. Besonders den ganz kleinen Patienten sollten eher Phytotherapeutika empfohlen werden. Sie sind nebenwirkungsarm und verfügen über eine lange Tradition und Anwendungserfahrung in der Arztpraxis - wie die Erkältungsmedizin von Eucabal ${ }^{\circledR}$. Durch ihre bronchospasmolytischen, sekretolytischen sowie antibakteriellen Eigenschaften sind sowohl der Hustensaft als auch der Erkältungsbalsam nahezu ideal für eine kindgerechte Atemwegstherapie.

Eucabal ${ }^{\oplus}$-Balsam S ist bereits für Kleinkinder ab sechs Monaten zur Rückeneinreibung zugelassen. Die darin enthaltenen Eukalyptus- und Kiefernnadelöle zeigen ein breites Wirkungsspektrum. Der Hauptbestandteil von Eukalyptusöl ist 1,8-Cineol (mind. $80 \%$ ). Dieses wirkt krampflösend, antibakteriell und schleimlösend. Die Hauptbestandteile der aus Kiefernnadeln gewonnenen ätherischen Öle - sogenannte a-Pinene - wirken hyperämisierend. So unterstützt Eucabal ${ }^{\circledR}$ Balsam S mit einem Anteil von $30-40 \%$ an
a-Pinen eine vertiefte Atmung. In Kombination verflüssigen und lösen die beiden ätherischen Öle das Bronchialsekret und sorgen für dessen Abtransport. Katarrhalische Reizerscheinungen im Respirationstrakt werden gelindert und ein Fortschreiten der Infektion wird gehemmt. In heißem Wasser aufgelöst, eignet sich die Erkältungscreme auch zur Inhalation, welche die genannten Effekte noch intensiviert - allerdings erst für Kinder ab sechs Jahre.

Eucabal ${ }^{\oplus}$-Erkältungsprodukte sind zwar klassische Arzneimittel für die Selbstmedikation, trotzdem stellen viele Kinderärzte ihren Patienten sowohl für den Hustensaft als auch für den Balsam, der bis zu einem Alter von zwölf Jahren sowieso erstattungsfähig ist, immer ein Rezept aus. Erstens können sie sich so sicher sein, dass die Eltern das richtige Arzneimittel in der Apotheke kaufen. Zweitens erstatten mittlerweile mehr und mehr Krankenkassen auch auf dem Grünen Rezept verordnete Phytotherapeutika.

Nach Informationen von Aristo

\section{Neurodermitis lindern ohne Kortison}

— In der Behandlung der Neurodermitis besitzen Cremes mit Glukokortikoiden nach wie vor einen hohen Stellenwert. Aber auch die begleitende, regelmäßige Hautpflege ist ein wichtiger Baustein, um Beschwerden nachhaltig zu lindern. Denn dies hilft, den Einsatz von Arzneimitteln auf ein Minimum zu reduzieren. Hierzu steht ab sofort mit Linola PLUS eine neuartige Kombination aus Linolsäuren und dem Wurzelextrakt aus der Echinacea-Pflanze (Purpur-Sonnenhut) als medizinische Hautpflege zur Verfügung. Gerade der Juckreiz kann so besser in Schach gehalten werden. Zudem kann der Entwicklung von Entzündungen vorgebeugt werden.

Der Dr.-Wolff-Forschung gelang es unter Verwendung eines besonderen Extraktionsverfahrens, die wertvollen Inhaltsstoffe aus der Echinacea-Pflanze, die traditionell bei Entzündungen der Schleimhäute im Nasen-Rachen-Bereich eingesetzt werden, auch für die medizinische Hautpflege nutz- bar zu machen und typische Beschwerden zu lindern. Denn der lipophile Wurzelextrakt enthält biologisch aktive Substanzen (u.a. Alkylamide), die juckreizlindernde und antientzündliche Eigenschaften aufweisen. Da im Extraktionsverfahren ausschließlich natürliche Quellkohlensäure verwendet wird, ist garantiert, dass im Gegensatz zu anderen Verfahren keine Lösungsmittelrückstände im Extrakt zurückbleiben. In diesem sogenannten "grünen Verfahren" verhindern milde Extraktionsbedingungen bei niedriger Temperatur gleichzeitig den Abbau der wertvollen Wurzelstoffe.

Linola PLUS wirkt zweifach: Die einzigartige Kombination aus Linolsäure-reichem Distelöl mit dem Wurzelextrakt der Echinacea purpurea unterstützt die Regeneration der Hautbarriere und gleichzeitig reduziert sie nachhaltig Juckreiz und Hautirritationen.

Nach Informationen von Dr. Wolff

\section{Vereinfachte Inhalationstherapie}

Mit Velox ${ }^{\circledast}$ und Velox ${ }^{\circledast}$ Junior erweitert PARI jetzt seine Produktpalette: Die beiden Inhalationsgeräte mit moderner Schwingmembran-Technologie sind optimal für die Therapie von akuten oder chronischen Atemwegserkrankungen bei Kindern und Erwachsenen geeignet. Die Patienten profitieren dabei von maximaler Mobilität durch den optionalen Batteriebetrieb, einfacher Handhabung, geringer Größe und geringem Gewicht (nur $110 \mathrm{~g}$ ohne Batterien) sowie einem nahezu geräuschlosem Betrieb. Der Velox ${ }^{\circledast}$ ist in zwei Ausstattungsvarianten in Apotheken und Sanitätshäusern erhältlich: Velox ${ }^{\oplus}$ inklusive Mundstück für Erwachsene und Kinder ab 3 Jahren und Velox ${ }^{\circledast}$ Junior inklusive weicher Smartmask ${ }^{\circledast}$ Baby und Mundstück für Babys und Kleinkinder bis 3 Jahre.

Nach Informationen von PARI

\section{Läuse-und Nissenkamm - so verordnen Sie ihn richtig}

Durch seine besonders eng stehenden und langen Metallzinken ermöglicht der NYDA ${ }^{\oplus}$ Läuse- und Nissenkamm eine zuverlässige Diagnose und Nachbehandlung bei Kopflausbefall. Der GKVSpitzenverband hat den Läuse- und Nissenkamm gemäß §139 SGB V ins Hilfsmittelverzeichnis aufgenommen und als verordnungsfähiges Hilfsmittel eingestuft - damit ist er derzeit die einzige verordnungsfähige Option in diesem Bereich. Eine Verordnung ist an die gleichzeitige Verschreibung eines Pedikulozids (wie z. B. des 2-Stufen-Dimeticons NYDA ${ }^{\oplus}$ ) auf einem separaten Rezept gebunden. Dann ist der Läuse- und Nissenkamm für Kinder unter 12 Jahren und Jugendliche mit Entwicklungsstörungen unter 18 Jahren verordnungsfähig. Um Rückfragen von Seiten der GKV von vorneherein zu vermeiden, gilt es bei der Verordnung folgendes zu beachten: Das Feld mit der Ziffer 7 für Hilfsmittel ankreuzen, die Hilfsmittelnummer 99.17.02.0001 angeben sowie zusätzlich zur Verschreibung des NYDA ${ }^{\oplus}$ Läuseund Nissenkamms die Diagnose und den Hinweis auf "gleichzeitige Verordnung eines Pedikulozids" vermerken. Wichtig zu wissen: Als Hilfsmittel kann der Kamm ohne Belastung des Praxisbudgets rezeptiert werden.

Nach Informationen von Pohl Boskamp 